\title{
Laparoscopic Resection of Bleeding Dieulafoy's Lesion in the Jejunum Following Intraoperative Localization Using Endoscopy
}

\author{
Hwan Hyo Lee, M.D. ${ }^{1}$, Seung Mook Jeong, M.D. ${ }^{2}$, Geon Min Kim, M.D. ${ }^{2}$, Ja Hong Ku, M.D. ${ }^{3}$ \\ Departments of ${ }^{1}$ Surgery, ${ }^{2}$ Internal Medicine, and ${ }^{3}$ Radiology, Gimpo Woori Hospital, Gimpo, Korea
}

\begin{abstract}
A Dieulafoy lesion is a relatively rare, but potentially life threatening cause of gastrointestinal bleeding, which accounts for only $1 \sim 2 \%$ of acute cases. The most common site is the stomach and most extragastric lesions are frequently found in the duodenum followed by the esophagus, the colon, and rectum. However, jejunum and ileum are extremely rare. I report on a case of a 38-year-old female who underwent laparoscopic resection of a bleeding Dieulafoy's lesion in the jejunum following intraoperative endoscopy.
\end{abstract}

Keywords: Dieulafoy, Jejunum, Intraoperative endoscopy, Laparoscopic resection
Received December 1, 2015

Revised December 29, 2015

Accepted February 15, 2016

Corresponding author

Hwan Hyo Lee

Department of Surgery, Gimpo

Woori Hospital, 389-15, Gulpo-

dong, Gimpo 10099, Korea

Tel: +82-31-999-1755

Fax: +82-31-999-1399

E-mail: corbrain@naver.com

In 33th congress of Korea

Endoscopic Laparoscopic Society, this title was presented as poster and received the top-best poster award.

Copyright (C) 2016 The Journal of Minimally Invasive Surgery. All rights reserved.

\section{INTRODUCTION}

A Dieulafoy's lesion is a relatively rare, but potentially life threatening cause of gastrointestinal bleeding, which accounts for only 1 $2 \%$ of acute cases. ${ }^{1,2}$ The most common site is stomach. Most extragastric lesions are frequently in the duodenum followed by the esophagus, the colon and rectum. ${ }^{2,3}$ However, jejunum and ileum are extremely rare, which accounts for only $1 \%$ of collected review cases. ${ }^{2,3}$ Bleeding Dieulafoy's lesion in the jejunum may be preoperatively diagnosed as angiography or computed tomography, but these modalities can not exactly preoperatively localized the bleeding site of small intestine. ${ }^{2,4-6}$ Intraoperative endoscopy can help exact localization of the Dieulafoy's lesion in small intestine during operation, which can be attributed to limit extent of resection and raise the accuracy of small bowel resection., ${ }^{2,47}$

\section{CASE REPORT}

A 38-year-old female presented to the emergency department complaining of abdominal pain and hematochezia. She had no past history or symptoms of gastrointestinal dysfunction. On emergency room, her blood pressure was 130/80 $\mathrm{mmHg}$, pulse rate $118 / \mathrm{min}$. She was very obese (body mass index: $40.66 \mathrm{~kg} / \mathrm{m}^{2}$ ). A physical examination of abdomen revealed a slightly distended abdomen and left upper quadrant tenderness. Rectal examination showed fresh blood clots. The WBC count was $8,650 / \mathrm{mm}^{3}$, hemoglobin level $10.4 \mathrm{~g} / \mathrm{dl}$, he- 
matocrit $30.1 \%$, platelet $384,000 / \mathrm{mm}^{3}$. Abdominal computed tomography (CT) was performed at emergency room and showed active bleeding in the jejunum and contrast leakage into jejunum (Fig. 1). On the night of admission, she suffered from several hematochezia and had a further episode of hypotension. At this time, her hemoglobin was $7.4 \mathrm{~g} / \mathrm{dl}$. Four

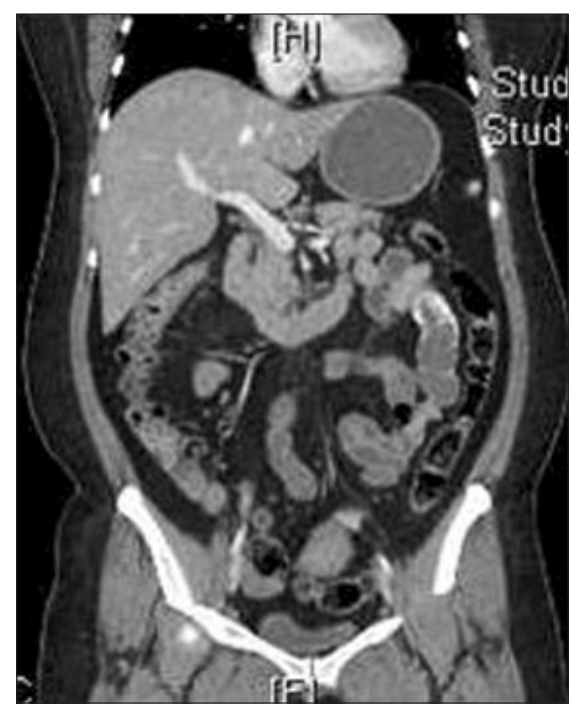

Fig. 1. Abdominal CT showed active bleeding in the jejunum and contrast leakage into jejunum. units of packed red cells were transfused at this night. Following day, superior mesenteric angiography was performed for localization and revealed active extravasation of contrast media from the 3rd branch of jejunal artery (Fig. 2). We tried to embolise with gelfoam and could decrease the amount of jejunal bleeding, but failed. The preoperative diagnosis was jejunal bleeding of unusual vascular malformation.

Emergent surgical exploration using laparoscopy was performed. Operative finding revealed that mesentery of proximal jejunum was several petechiae and bowel loops filled with hematoma. We could not localized bleeding site. Proximal jejunal loop with mesenteric petechiae was brought out abdominal wall through small incision. Intraoperative endoscopy was performed for localization of bleeding site and identification of its cause through a jejunotomy, and showed bloody oozing from $6 \mathrm{~mm}$ sized elevated submucosal lesion with central mucosal defect and small exposed vessel at jejunal loop $40 \mathrm{~cm}$ distal to Treitz ligament (Fig. 3). We performed laparoscopic assisted segmental resection of this segment. Pathological examination confirmed the diagnosis of Dieulafoy's lesion in the jejunum. The lesion consisted of a small mucosa erosion with a large tortuous submucosal vessel filled with thrombi (Fig. 4). Postoperatively, the patient had an uneventful recovery without gastrointestinal bleeding. She was discharged on postoperative day 9 and reported no further bleeding episode at a 24-month follow-up visit.
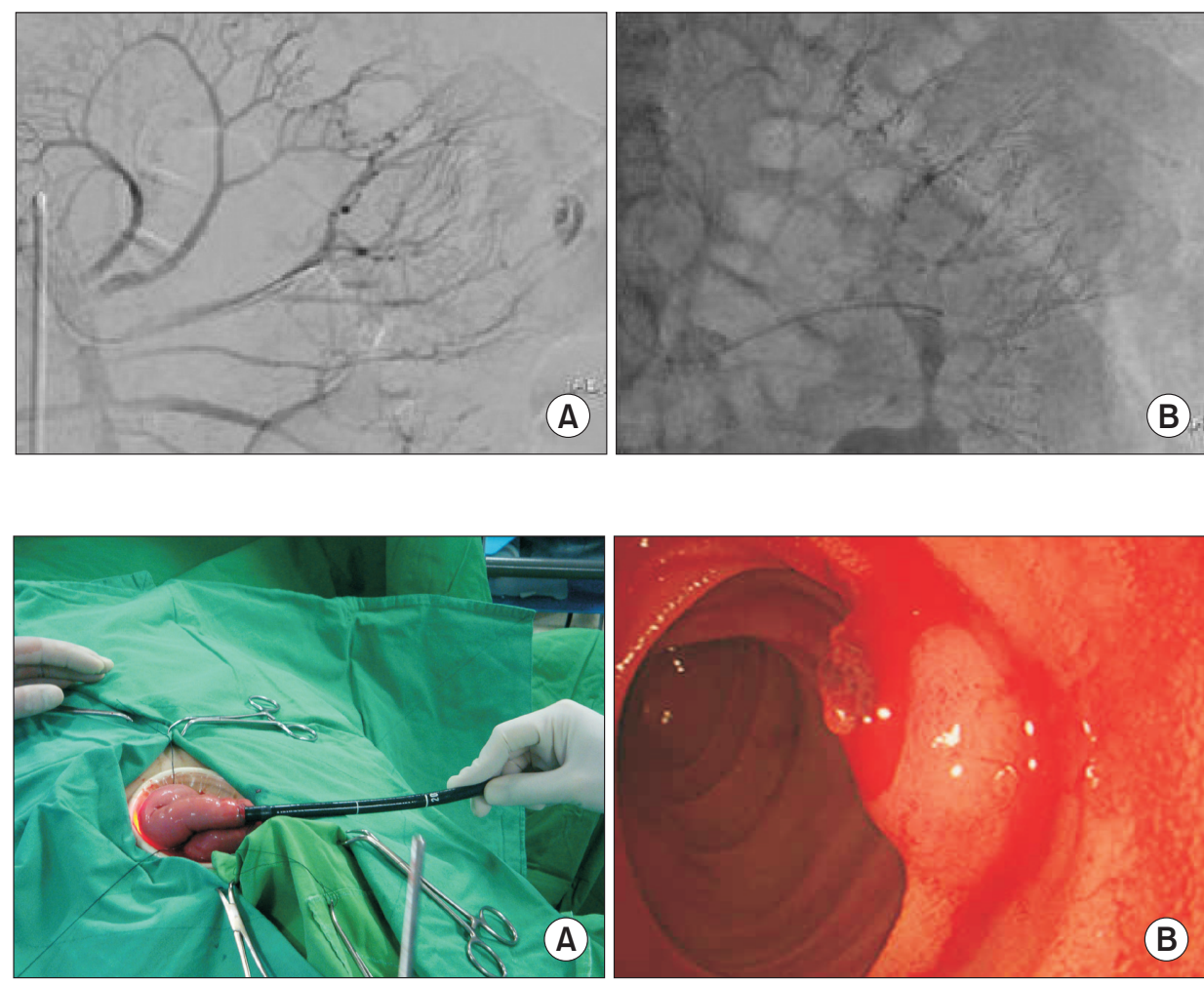

Fig. 2. (A) Superior mesenteric angiography showed active extravasation of contrast media from the 3rd branch of the jejunal artery. (B) After embolization, the amount of jejunal bleeding is decreased.

Fig. 3. (A) Intraoperative endoscopy was performed through a jejunostomy. (B) Endoscopic finding showed bloody oozing from $6 \mathrm{~mm}$ sized elevated submucosal lesion with central mucosal defect and small exposed vessel. 


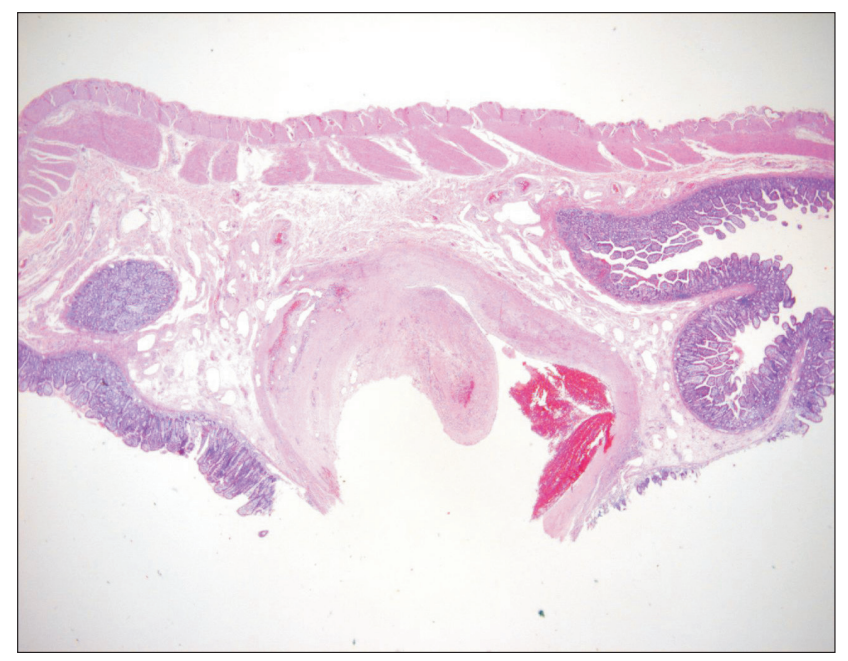

Fig. 4. Histopathological examination (H\&E stain, $\times 125)$ showed a small mucosa erosion with a large tortuous submucosal vessel filled with thrombi.

\section{DISCUSSION}

A Dieulafoy's lesion is an aberrantly dilated and tortuous submucosal artery, often identified after it erodes through the overlying mucosa of the gastrointestinal tract and begins to rupture. Histologically, a superficial mucosal defect, or erosion without ulcer sign is present in the mucosa overlying the artery with focal necrosis and rupture of the artery at the base of the erosion. Bleeding occurs from a small mucosal erosion involging an unusually large submucosal artery. ${ }^{1-3}$

The most common site of Dieulafoy's lesion is stomach, accounting about $71 \%$. Approximatly one-third of lesions are extragastric, most frequently in the duodenum (15\%) followed by the esophagus (8\%), and the colorectum (4\%). ${ }^{2,3}$ It has very rarely described in small intestine $(1 \%)^{2}$

Dieulafoy's lesions typically present suddenly as massive hemorrhage which is often recurrent and can take the form of hematemesis, melena or hematochezia. Most patients show unstable vital sign and need transfusion before operation. ${ }^{1-4}$

At present, the diagnosis of Dieulafoy's lesion is effectively made by endoscopy, up to 70 80\%, although repeated endoscopy was required in some cases. ${ }^{1-3}$ The assessment of the small intestine could be done by push enteroscopy, intraoperative enteroscopy or capsule endoscopy. ${ }^{1-3}$ The characteristic endoscopic findings of a Dieulafoy's lesion in the small intestine are identical to gastric lesion: 1) active arterial spurting or micropulsatile streaming from a mucosal defect (less than 3 $\mathrm{mm}$ ) or through normal surrounding mucosa; 2) visualization of a protruding vessel with or without bleeding, within a minute mucosal defect or through normal surrounding mucosa; 3) the appearance of fresh, densely adherent clot with a narrow point of attachment to a minute mucosal defect or to normal appearing mucosa.

Angiography can be useful to locate the source of bleeding that can not be diagnosed by endoscopic methods or during haemodymical unstability due to massive bleeding. ${ }^{1,2,5,6}$ The findings of angiography that suggested Dieulafoy's lesion include a tortuous and ectatic artery and contrast extravasation from a normal looking blood vessel. ${ }^{5}$ Also, CT angiography may assume a rapid, noninvasive diagnostic role in suspected acute gastrointestinal bleeding, although its role seems to remain limited as therapeutic modality.,

Theatment modalities of Dieulafoy's lesion are dependent on mode of clinical presentation, site of lesion and available medical teams involving endoscopists, interventional radiologists, and surgeons. ${ }^{1,2,4}$ The evolution of endoscopic methods of haemostasis has markedly reduced the need for surgery. ${ }^{1-3}$ Endoscopic methods are the treatment of choice in easily accessible lesions, with success rates over $90 \%{ }^{1-3}$ These procedures can be classified into three groups: 1) thermal - electrocoagulation, agron plasma coagulation; 2) regional injection - epinephrine, sclerant; 3) mechanical - endoscopic band ligation, haemoclips. More studies have shown that endoscopic mechanical treatments are more effective and successful in achieving haemostasis than other endoscopic methods. ${ }^{1-3}$ Also, angiography with selective embolization can be useful to control actively bleeding Dieulafoy's lesion with following situations: 1) lesions that fail to respond to endoscopic therapy; 2) lower GI bleeding or lesions beyonds reach of therapeutic endoscopy; 3) poor candidates for surgery. ${ }^{1,2,4}$ Also, this method may aid in slowing down hemorrhage, thereby allowing more time for further resuscitative efforts in preparation for surgery. ${ }^{4}$ Surgical resection has today been overtaken by advances in endoscopic procedures. Actually, surgery has a role in the 5\% of cases that are failed to endoscopic or angiographic methods. ${ }^{1,2}$ Surgical procedures employed include a wedge resection of involved segment of gut or under-running sutures of the lesion. Recently, laparoscopic surgery is an attractive option for these lesions. Some authors reported cases describing successful laparoscopic wedge resection of bleeding Dieulafoy's lesions in the jejunum. ${ }^{9}$ However, the success of laparoscopic surgery depends on accurate localization of the bleeding site. Intraoperative localization includes palpation, identification of the most proximal site of intraluminal blood, transilumination of the small bowel wall, and enterotomy or endoscopy through enterotomy. ${ }^{2,4,7,8,10}$ For accurate intraoperative localization of these lesions, several methods using intraoperative endoscopy have been described. ${ }^{2,4,7,10}$ Intraoperative enteroscopy was the gold standard for detection of lesions in the small bowel, allowing the exploration of the entire mucosa. ${ }^{4}$ As in the case of 
obscure GI bleeding, small bowel endoscopy can be accomplished intraoperatively where it will involve a team comprised of a surgeon and an endoscopist. Intraoperative enteroscopy via small bowel enterotomy should be undertaken to dictate the extent of resection and lessen the chance for rebleeding.

In conclusion, we consider accurate intraoperative localization using endoscopy may allow limited resection of small bowel and lower rebleeding rates. In a case with bleeding Dieulafoy's lesion in the jejunum, laparoscopic resection using intraoperative endoscopy is a feasible and safe alternative to conventional open method.

\section{REFERENCES}

1) Chaer RA, Helton WS. Dieulafoy's disease. J Am Coll Surg 2003; 196:290-296.

2) Baxter M, Aly EH. Dieulafoy's lesion: current trends in diagnosis and management. Ann R Coll Surg Engl 2010;92:548-554.

3) Nojkov B, Cappell MS. Gastrointestinal bleeding from Dieulafoy's lesion: Clinical presentation, endoscopic findings, and endoscopic therapy. World J Gastrointest Endosc 2015;7:295-307.

4) Goins WA, Chatman DM, Kaviani MJ. Massive lower gastroin- testinal bleeding due to 'Dieulafoy's vascular malformation' of the jejunum: case report. J Natl Med Assoc 1995;87:766-770.

5) Durham JD, Kumpe DA, Rothbarth LJ, Van Stiegmann G. Dieulafoy disease: arteriographic findings and treatment. Radiology 1990;174:937-941.

6) Cherian MP, Mehta P, Kalyanpur TM, Hedgire SS, Narsinghpura KS. Arterial interventions in gastrointestinal bleeding. Semin Intervent Radiol 2009;26:184-196.

7) Monsanto P, Almeida N, Lerias C, Figueiredo P, Gouveia H, Sofia C. Is there still a role for intraoperative enteroscopy in patients with obscure gastrointestinal bleeding? Rev Esp Enferm Dig 2012; 104:190-196.

8) al-Mishlab T, Amin AM, Ellul JP. Dieulafoy's lesion: an obscure cause of GI bleeding. J R Coll Surg Edinb 1999;44:222-225.

9) Mino A, Ogawa Y, Ishikawa T, et al. Dieulafoy's vascular malformation of the jejunum: first case report of laparoscopic treatment. J Gastroenterol 2004;39:375-378.

10) Blecker D, Bansal M, Zimmerman RL, et al. Dieulafoy's lesion of the small bowel causing massive gastrointestinal bleeding: two case reports and literature review. Am J Gastroenterol 2001;96:902905. 